\title{
Correspondence
}

\section{Growth and childhood asthma}

Sir,

We read the recent article by Dr Balfour-Lynn with interest. ${ }^{1}$ In this study the prepubertal height centile from a 'distance' chart of each individual child was extrapolated to the adult centile as an assessment of final height prognosis. It is the degree of epiphysial maturation, however, that determines the proportion of growth that has occurred and hence the height potential remaining; only $40 \%$ of the children in this study had an assessment of bone age. A further assumption was the use of development of pubic hair as a sign of the onset of puberty. Pubic hair, which is predominantly associated with adrenal androgen secretion, is not necessarily a sign of the onset of puberty. Luxurious development of pubic hair is common in children with absent puberty secondary to hypogonadotrophic hypogonadism or gonadal dysgenesis.

Figure 1 of this article depicted the timing of the growth spurt of a boy with asthma coincident with the onset of puberty. One of the characteristics of puberty in boys is that the onset of the spontaneous growth acceleration occurs late in puberty, between genitalia stage 3 and $4^{2}$ or more accurately at the attainment of a $10 \mathrm{ml}$ testicular volume. Clearly, in Figure 1 the growth spurt was either unrelated to the spontaneous growth acceleration of puberty and perhaps due to 'catch up' growth secondary to effective treatment or the assessment of the onset of pubertal maturation was incorrect. If the former was correct then the growth pattern was not due to delayed puberty; the latter would put the accuracy of the entire study into doubt. The data presented showed a loss of the harmony of normal growth and puberty, and this usually points to an endocrine abnormality. ${ }^{3}$

Such a longitudinal study should be an important contribution to our understanding of the growth of children with asthma, many of whom have delayed growth and puberty. The data presented and the errors in methodology, however, do not permit the conclusions that Dr Balfour-Lynn has made.

\section{$\mathrm{R}$ Stanhope and $\mathrm{M}$ A Preece Institute of Child Health, London WCI}

\section{Dr Balfour-Lynn comments:}

It is intriguing to learn from the department made famous by Professor Tanner that children who faithfully follow one of the centile lines on his growth and development charts throughout the whole of their growing life cannot be considered to have grown normally. In this study all 66 children were directly observed to do this up to at least 10 years of age, during the most severe period of their childhood asthma. Thirty five children continued to follow their chosen centile, whereas 30 were observed to have delay in the onset of puberty with a typical growth delay curve, even though in many this coincided with an improvement in their asthma and some, like the child in Figure 1, were actually free of symptoms. Satisfactory catch up growth occurred in all these children. If this does not mean that asthma has no direct effect on growth, apart from indirectly by causing pubertal delay, then Professor Tanner's charts should no longer be considered acceptable standards and should be scrapped in every paediatric clinic in the country. Support for this study was found, however, in $40 \%$ of these children from bone age predictions, a percentage highly acceptable to statisticians if not to Professor Preece and Dr Stanhope. Moreover, support was also presented from $91 \%$ of the parental heights, a fact ignored by them.

Pubic hair was mentioned in the assessment of puberty as Professor Tanner included it in great detail in all his charts. ${ }^{2}$ It was not noted to be the first sign of puberty in any of the boys, but it was in two girls. If this is not acceptable as a sign of the onset of puberty then it means that the delay was even greater in the girls in this study.

It is absurd to suggest that all this consistent data is destroyed by the chart of one boy. He had the greatest delay in the onset of puberty of all the children in the study and consequently the greatest centile loss in height during the deceleration in growth velocity. Thus his pubertal delay curve was used as it was the most dramatic, though possibly some smoothing off by the medical artist has caused a little confusion. His growth curve was especially interesting as he was one of the milder cases of asthma. He was successfully treated with regular sodium cromoglycate from the age of 7 to 11 years, after which he rarely needed to use a salbutamol inhaler and was soon entirely free of symptoms. There is no way his growth delay could be directly attributable to asthma or treatment. Because of his pronounced pubertal delay, however, he was carefully observed as his parents were by then more concerned with this than his asthma. Changes were first detected in his testes when seen at the age of 15.8 years (accuracy to within six months), which were obvious six months later. When he was aged 16.8 years his genitalia had reached stage 3 and pubic hair was first noted. A growth velocity curve shows that his peak height velocity coincides with this stage. Marshall and Tanner, in the paper quoted by Professor Preece and Dr Stanhope, noted peak height velocity at stage 3 of puberty, though it occurred more commonly at stage $4 .^{2}$ Marshall and Tanner, however, were recording a series of children with normal growth, but in my experience of pubertal growth delay the greater the delay the more dramatic and earlier the growth spurt once puberty develops.

In this study the methodology used for the assessment of growth, puberty, and bone age prediction have relied heavily on the work of Professor Tanner. It is surprising that this is no longer acceptable to his old department. 
References

1 Balfour-Lynn L. Growth and childhood asthma. Arch Dis Child 1986;61:1049-55.

2 Marshall WA, Tanner JM. Variations in the pattern of pubertal changes in boys. Arch Dis Child 1970;45:13-23.

3 Stanhope R, Brook CGD. The clinical diagnosis of disorders of puberty: the loss of consonance. BrJ Hosp Med 1986;35:57-8

\section{Screening for the detection of congenital dislocation of the hip}

Sir,

We have read with interest the special report on 'Screening for the detection of congenital dislocation of the hip' prepared by an advisory committee for the Secretary of State. ${ }^{1}$ It is recommended that the Ortolani/Barlow manoeuvre be carried out within 24 hours of birth; first the Ortolani procedure then the Barlow manoeuvre, 'with the thumb on the inner side of the thigh, backward pressure is applied to the head of the femur,' if a 'clunk' is obtained, 'the head is said to be subluxatable (dislocatable)'. In other words, the hip is dislocated.

One of us ( $\mathrm{CHC})$ has noticed increasing laxity in the hip joint of an infant who has been repeatedly examined to show physical signs to junior medical colleagues. In view of the risks associated with 'excessive manipulation of the hip joint', the advisory committee recommend that 'duplication of the examination by both midwife and doctor should be avoided. Each maternity unit should determine its own policy in this respect to ensure that there is only one examination.' We submit that this advice is unrealistic and that in practice a midwife who discovers that she can dislocate a hip will continue to ask the resident doctor to confirm her finding and this will be checked by a paediatric registrar or consultant, or both. The orthopaedic surgeon will probably carry out a further examination before applying a splint for what has become a recurrent dislocation.

The Southampton experience ${ }^{2}$ of an increasing incidence of late congenital dislocation in an area practising enthusiastic neonatal screening with a reluctance to undertake unnecessary early splinting leads us to the conclusion that Barlow's manoeuvre in the first two days of life was converting normal joint laxity into established dislocation.

Barlow's manoeuvre does not distinguish between a normal hip and one that untreated will develop an established dislocation. Barlow showed that dislocatable hips are much more common in the first three days than later in the first week, and no cases of late dislocation were discovered in his infants who were first examined after the age of 3 days. $^{3}$

We consider that during the first 48 hours of life when ligamentous laxity is so common great care should be taken not to dislocate a baby's hips either by swaddling with adducted thighs or by Barlow's manoeuvre. At this time clinical examination should be directed to discovering established dislocation by looking for asymmetry, apparent shortening of the femur, restriction of abduction, and a 'clunk' by the Ortolani procedure.

We welcome the recommendation that repeated ex- aminations for dislocation of the hip should be carried out throughout infancy because the evidence indicates that some dislocations are undiagnosable at birth and develop later.

C H Cheetham and D H Garrow Wycombe General Hospital, High Wycombe, Bucks HP11 2TT, and Roughwood Farmhouse, Chalfont St Giles, Bucks

Sir,

It has always been my impression that the chance of detecting congenital dislocation of the hip would be increased if the number of examinations by an experienced observer were increased. In the recent article on congenital dislocation of the hip it is stated, 'In view of the risks associated with excessive manipulation of the hip joint, duplication of the examination by both midwife and doctor should be avoided.'

What is the scientific evidence that there are such risks, and if there is indeed a risk what is its incidence?

I Blumenthal

Oldham and District General Hospital, Oldham OLI $2 \mathrm{JH}$

\begin{abstract}
References
1 Standing Medical Advisory Committee and Standing Nursing and Midwifery Advisory Committee Working Party. Screening for the detection of congenital dislocation of the hip. Arch Dis Child 1986;61:921-6.

2 Catford JC, Bennett GC, Wilkinson JA. Congenital hip dislocation: an increasing and still uncontrolled disability. $\mathrm{Br}$ Med J 1982;285:1527-30.

${ }^{3}$ Barlow TG. Early diagnosis and treatment of congenital dislocation of the hip. J Bone Joint Surg [BR] 1962;48:292-301.
\end{abstract}

\section{Dr Dunn comments:}

Thank you for inviting me as a member of the SMAC/ SNMAC working party under the chairmanship of Professor E Stroud to respond to these letters.

Drs Cheetham and Garrow's first point deals with a semantic problem. Most abnormal hips at birth are of the unstable, subluxatable variety. They can be partially dislocated by backward pressure when the thigh is adducted and flexed. We included the word 'dislocatable' in parenthesis because this term is in common use, even though such hips do not fully dislocate, as may be shown at necropsy.

Next they question our exhortation to avoid excessive manipulation of the hip joint, a point also raised by $\mathrm{Dr}$ Blumenthal. The working party thought it important to emphasise that repeated examination might damage an unstable hip. While there is no clinical evidence to support or refute this belief, manipulation of the unstable hip at necropsy seems to increase hip instability. The artery in the ligamentum teres is also vulnerable to trauma. Of course, it was appreciated that some repetition of hip examination was necessary as part of the ongoing screening process as well as to confirm the diagnosis in suspected cases. 